\title{
Alto y bajo: de lo dimensional a lo cualitativo. Propuesta lexicográfica
}

\author{
Carlos Ynduráin \\ University of Namibia \\ carlosyndurain@gmail.com \\ https://dx.doi.org.10.12795/futhark.2020.i15.13
}

Fecha de recepción: 18.04 .2020

Fecha de aceptación: 25.06 .2020

Resumen: En torno a los valores prototípicos de los adjetivos alto y bajo (relacionados, en principio, con las dimensiones de una entidad física) existe una serie de significados de origen metafórico que vinculan estas palabras con los conceptos de CANTIDAD (altas dosis), INTENSIDAD (alta tensión) y FRECUENCIA (ventas altas). El objetivo de este artículo es analizar la base cognitiva de estas extensiones semánticas y clasificar los sentidos que resulta apropiado diferenciar de cara a su posterior tratamiento lexicográfico.

Palabras clave: adjetivos; adverbios; adjetivos dimensionales; metáfora; metonimia.

\section{The adjectives alto ('tall', 'high') and bajo ('short', 'low'): from dimensional to quantitative meaning. Lexicographic proposal}

\begin{abstract}
Regarding the prototypical value of Spanish adjectives alto and bajo (originally related to the spatial dimensions of an entity), there is a series of meanings, metaphorical in origin, that link these terms with the concepts of QUANTITY (altas dosis), INTENSITY (alta tensión) and FREQUENCY (ventas altas). The aim of this article is to analyze the cognitive basis of these semantic extensions and to classify the meanings that are relevant for their subsequent lexicographic treatment.
\end{abstract}

Keywords: adjectives; adverbs; dimensional adjectives; metaphor; metonymy. 
Sumario: I. Introducción. 2. Alto y bajo. significados prototípicos. 2.I. Alto y bajo. ubicación. 3. Extensión metafórica hacia cantidad, intensidad y frecuencia. 4. Propiedades y eventos. 4.I. Usos relacionados con las propiedades. 4.2. Usos relacionados con los eventos. 5. Explicación de la propuesta lexicográfica. 5.I. Estructura de la propuesta. 5.2. Separación y agrupamiento de acepciones. 5.3. Las definiciones. 5.3.I. "Lo normal 0 adecuado". 5.4. Cuestiones relativas a cada acepción. 5.4.I. Adjetivos. 5.4.2. Adverbios. 6. Propuesta lexicográfica.

\section{Introducción}

Los adjetivos alto y bajo presentan una amplia red polisémica en torno a sus significados prototípicos, es decir, en torno a aquellos significados que se relacionan con las propiedades dimensionales de los objetos.

La polisemia, a pesar de que pueda registrarse y rastrearse lingüísticamente, es, en esencia, un fenómeno mental: los múltiples significados que presentan las palabras son el reflejo tangible de mecanismos cognitivos universales. Nuestra intención es describir y explicar los mecanismos concretos que subyacen a unas extensiones semánticas específicas: aquellas en las que los adjetivos mencionados (alto y bajo) se relacionan con los conceptos de CANTIDAD/FRECUENCIA e INTENSIDAD.

Asociaciones como altas dosis, alta tensión o ventas altas son una muestra de cómo estos adjetivos, en principio dimensionales', se vinculan con nombres que hacen referencia a realidades que, en sentido estricto, no presentan un TAMAÑO físico y que, por lo tanto, no son susceptibles de ser calificadas dimensionalmente.

Al tiempo que abordamos el origen de estas metáforas conceptuales, trataremos también de aislar ( $y$ agrupar) los diferentes sentidos figurados que nos ocupan en una propuesta de carácter lexicográfico que contribuya a la sistematización de su tratamiento.

\section{Alto y bajo: significados prototípicos}

Podemos decir que alto y bajo presentan tres interpretaciones básicas relacionadas con la dimensión de los objetos, dependiendo de si estos adjetivos se conciben desde una perspectiva absoluta, intrínseca o relativa ${ }^{2}$.

\footnotetext{
' Los adjetivos dimensionales hacen referencia prototípicamente a las magnitudes de un objeto en el espacio: «En cuanto a los adjetivos dimensionales, el término 'dimensión' denota principalmente las tres dimensiones espaciales de los objetos físicos, a saber, la largura/altitud, la anchura y el volumen o profundidad» (Mulier 2009: 12).

${ }^{2}$ Esta distinción se basa en la clasificación establecida por Levinson (2003) para las relaciones espaciales entre una figura y un fondo, así como en su adaptación para la concepción de las propiedades dimensionales de los objetos tomados individualmente (Ynduráin 20I5: 212-226).
} 
Desde una perspectiva intrínseca, la ALTURA de un objeto es la dimensión vertical que presenta cuando se encuentra en su posición de equilibrio canónica. Así, aunque se 'tumbe' un armario para su transporte, se puede decir de este que es alto o bajo sin hacer referencia al espacio vertical que ocupa al ser transportado, sino al que ocuparía si estuviera en uso.

Desde una perspectiva relativa, la atribución de una ALTURA a un objeto depende de la forma de este, es decir, de los tamaños relativos de sus distintas partes. Por analogía con las proporciones humanas, en los objetos que carecen de una orientación vertical canónica, es frecuente considerar que la ALTURA es la mayor de sus tres dimensiones básicas ${ }^{3}$.

Desde una perspectiva absoluta, la ALTURA coincide con la dimensión de un objeto sobre el eje vertical. En nuestra cultura no disponemos de ningún eje horizontal marcado al que podamos tener acceso de forma intuitiva. El único eje incorporado a nuestro día a día es el paralelo a la fuerza de la gravedad: el eje vertical. Este eje posee, además, como señala Langacker $(1987)^{4}$, una clara direccionalidad (marcada por la propia gravedad), por lo que siempre resulta accesible la distinción ARRIBA/ABAJO a la hora de diferenciar (a través de un criterio absoluto) las partes (superior e inferior) de un objeto.

There is simply nothing like the reliably fixed axis of the vertical to be found on the horizontal. In a few parts of the world, there may be strong environmentally determined axes [...] but these are exceptional cases. (Levinson 2003: 76)

La referencia a la altura absoluta de los objetos permite también ubicarlos respecto a una superficie que puede asimilarse al 'punto cero' de un eje de coordenadas: «Gravity defines a «natural direction», verticality, and a plane of reference, the ground level» (Clark 1973: 32-33). La altura absoluta de los objetos se asocia, por lo tanto, con la idea de CRECIMIENTO OPUESTO A LA FUERZA DE LA GRAVEDAD a partir de una BASE o PUNTO DE APOYO ${ }^{5}$ que actúa como referencia.

\footnotetext{
3 También puede entenderse como la LONGITUD, si la mayor de las dimensiones básicas es claramente preponderante (Ynduráin 2015: 219).

${ }^{4}$ Citado, en este caso, a través de Vogel (2004: 62).

${ }^{5}$ Estas características provocan, tal y como se recoge en Dirven y Taylor (1988: 392-393), que resulte extraña la asociación estalactita alta, a pesar de que las estalactitas muestren una orientación vertical claramente marcada; al tratarse de objetos que crecen en la dirección de la gravedad (y que no cuentan con una base que les sirva de punto de apoyo), su dimensión vertical no se asocia nunca con concepto ALTURA, sino con el de LONGITUD: las estalactitas son más o menos largas o cortas. Vogel (2004: 62) recoge otros ejemplos similares: «Geckeler (1997) suggests that an argument for the direction of French haut 'high' (from bottom to top) is that a thread hanging from the ceiling will not be called haut 'high' but long 'long'. Linde-Usiekniewicz (2000) encompasses the same phenomena by stating that objects to which HEIGHT is assigned are directed upwards. Therefore, neither curtains, nor lamps hanging on a rigid

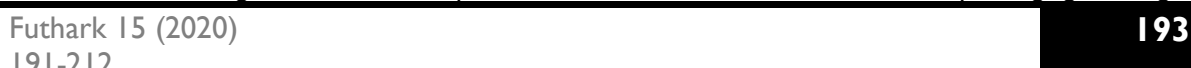




\section{I. Alto y bajo: ubicación}

Más allá de los usos estrictamente dimensionales, los adjetivos alto y bajo cuentan también con un significado relativo a la altura a la que se sitúan los objetos (o los eventos); es decir, con un significado relativo a la ubicación de estos: «El sintagma una cueva alta [...] puede designar una cueva situada en la parte alta de una montaña, o bien, una cueva en la que la distancia entre el suelo y el techo es considerable» (Bosque 1985: 69). La primera de las interpretaciones de Bosque haría referencia a la ubicación de la cueva. La segunda, a una de sus dimensiones.

En techos altos o en nubes bajas tampoco se hace referencia a la altura que presentan estas entidades, sino a la posición en que se ubican dentro del eje vertical. La distancia que separa a un objeto del punto que actúa como referencia (el suelo de una casa, la superficie terrestre...) se concibe como una entidad a la que es posible atribuir una altura. El adjetivo, sin embargo, se relaciona directamente con nombre del objeto, indicando, a través de un proceso metonímico evidente, que la línea imaginaria que separa a dicho objeto del eje horizontal es más o menos alta.

En términos generales puede decirse que en la metonimia una entidad sustituye (en lo lingüístico o en lo mental) a otra entidad: a través de la referencia a aquello que se considera prominente y fácilmente codificable se evoca lo que resulta cognitivamente menos accesible.

La mayor o menor prominencia de las entidades depende de su capacidad para atraer nuestra atención: «by virtue of their very nature, some entities are better qualified to attract our attention than others and are thus more salient in this sense» (Schmid 2007: 120). Para aproximarnos al concepto de ENTIDAD PROMINENTE resulta especialmente relevante la siguiente aportación de Benczes (2006):

Langacker (1993: 30; also see Radden and Kövecses 1999) explains that there are three main principles that govern our selection of an entity as being salient: human experience [...], perceptual [...] and cultural [...] factors. Radden and Kövecses (1999: 45) account for these preferences by our anthropocentric view of and interaction with the world. Accordingly, humans take precedence

pole from the ceiling are HIGH». Vogel (2004: 67) apunta, además, que, en esta línea «Rakhilina (2000) claims that objects, which are attached at some other object, and directed downwards, are not described as Russian vysokij 'high'». Para el caso del español, Bosque (1985: 76) señala que «los adjetivos corto y largo no remiten necesariamente a la dimensión horizontal (piénsese en un vestido largo)». Corrales Zumbado (1977: 64-65) señala, respecto a los términos que denotan dimensión vertical, que «el sentido es también un sema diferenciador a tener en cuenta [...]. De ahí que sea necesario separar en dos grupos las palabras según sea 'sentido hacia arriba' o 'sentido hacia abajo' lo que expresa la lexía considerada». 
over non-humans, things are viewed from a subjective rather than an objective point of view, concrete objects are regarded as cognitively more salient than abstract entities, things we interact with are chosen over things we do not interact with, and functional things are more important than non-functional things. (Benczes 2006: 52)

En los casos en los que los adjetivos dimensionales alto y bajo indican la ubicación de un objeto, podemos decir que la base de la metonimia se encuentra en el hecho de que las distancias que separan a los objetos de aquello que actúa como referencia (la superficie terrestre o el suelo, habitualmente) no son realidades sólidas y tangibles sobre las que resulte sencillo focalizar la atención. Los objetos situados a determinada altura, sin embargo, sí resultan fácilmente identificables y presentan, creemos, una mayor inmediatez cognitiva.

[...] an expression's usual referent (i.e. its profile) is invoked as a reference point to stablish mental contact with its intended referent (the target). To serve to this purpose effectively, $R$ has to be salient with respect to $T$. (Langacker 1995: 28)

Además, las referencias a las distancias resultarían lingüísticamente más complejas que vincular directamente el adjetivo dimensional y el nombre del objeto que se ubica a determinada altura: iun techo alto equivaldría a un techo situado a gran altura? ¿Una nube baja sería una nube que está a poca distancia del suelo? Consideramos, pues, que la asociación directa entre los adjetivos y los nombres de los objetos responde también a la búsqueda de un método sencillo de codificación lingüística.

\section{Extensión metafórica hacia CANTIDAD, INTENSIDAD y FRECUENCIA}

Una de las características de la interacción entre el hombre y su entorno es que ciertos tipos de experiencia están frecuentemente correlacionados, es decir, la aparición de uno de los fenómenos lleva consigo la presencia del otro y, en nuestro sistema conceptual, tienden a identificarse.

Esto es lo que subyace a la asociación mental (y lingüística, como veremos) de ARRIBA con MÁS O MUCHO y de ABAJO con MENOS O POCO: «if you add more of a substance or of physical objects to a container or pile, the level goes up» (Lakoff 1980:16). Esta elevación vertical se da de forma recurrente cuando se produce un aumento en la cantidad de cualquier substancia o grupo de objetos, por ello tendemos a identificar los dos fenómenos, y la idea de MÁS CANTIDAD 6 se vincula

\footnotetext{
${ }^{6}$ Santos Domínguez y Espinosa Elorza (1996: 56) señalan que, en español, la propia palabra suma pone de manifiesto que la operación a la que hace referencia se concibe como un amontonamiento de entidades, pues proviene de summam 'lo más alto'.
} 
con la idea de MÁs ALTURA ${ }^{7}$. La fuerza de este vínculo mental se hace patente en el lenguaje: con frecuencia se evocan las ideas de ALTO/ARRIBA/ELEVACIÓN Y BAJO/ABAJO/DESCENSO para hacer referencia a propiedades y cambios cuantitativos en entidades que no son de carácter físico, y que, por lo tanto, no pueden desplazarse por el eje vertical ni presentar dimensiones. En expresiones como presupuesto alto o ventas bajas $^{8}$ las referencias al eje vertical presentan un valor puramente metafórico.

Esta metáfora se basa en la activación metonímica de la noción de cantidad mediante la noción de un grado de verticalidad, debido a su frecuente asociación experiencial. [...] Esta reiterada conexión experiencial entre verticalidad (altura) y cantidad lleva a una conexión abstracta - metafóricaentre ellas, en contextos en los que verticalidad y cantidad ya no están realmente asociadas. (Barcelona 2012: 135)

A pesar de que «not every language has a MORE IS UP metaphor» - (Lakoff 1993: 35)-, dicha asociación se asienta en una base experiencial subjetiva que hace de ella, como señala Kovecses (2015: 6), una metáfora primaria9.

Las metáforas cognitivas permiten conceptualizar de forma sencilla elementos cuya comprensión resulta poco intuitiva: posibilitan la concepción de un tipo de realidades en términos de otras, a través de sus características comunes. Los conceptos primarios sobre los que construir el entramado metafórico en que vivimos nos los proporciona el contacto sensorial con el mundo físico: todo el sistema conceptual humano es, en última instancia, el producto de nuestra interacción con el entorno físico en que habitamos.

[...] the semantic representation coded by language reflects conceptual structure. If the embodiment of experience indeed gives rise to meaning, which is to say, conceptual structure, then the concepts expressed by language

\footnotetext{
${ }^{7}$ Para expresar cuestiones relativas a la CANTIDAD es también frecuente la metáfora «LINEAR SCALES ARE PATHS». Como señala Lakoff (1993: 2), «[w]e can see this in expressions like: John is far more intelligent than Bill. John's intelligence goes way beyond Bill's. John is way ahead of Bill in intelligence. The metaphor maps the starting point of the path onto the bottom of the scale and maps distance traveled onto quantity in general. What is particularly interesting is that the logic of paths maps onto the logic of linear scales». En español, esta es también la metáfora que subyace a Las ventas avanzan o Las ventas retroceden. Esta concepción metafórica no implica, sin embargo, el empleo de adjetivos del tipo largo/corto. Sí encontramos algunos usos de largo (largos intentos, largas tardes...) en los que el adjetivo puede relacionarse con el concepto de FRECUENCIA (Ynduráin 2019: 35).

${ }^{8}$ Bosque (1985: 67) señala que «el adjetivo inglés high y el alemán hoch poseen una productividad mayor que nuestro alto, y su uso, menos literario, los ha convertido en simples adjetivos de grado».

9 La 'fuerza' de esta metáfora puede constatarse en el hecho de que, aunque no todas las lenguas expresen la relación señalada, ninguna presenta la relación contraria: «There are other languages in which MORE IS UP and LESS IS DOWN, but none in which the reverse is true» (Lakoff 1993: 34).
} 
should largely derive from our perception of spatio-physical experience. [...] Spatio-physical experience provides much of the fundamental semantic (or conceptual) structure from which other concepts are constructed. (Tyler y Evans 2003: 24)

If a primary metaphor is the basis for a given metaphorical blend, this is certainly only the beginning of a process. Primary metaphors constitute counterpart connections which ultimately live inside of larger conceptual complexes. (Grady 2005; 1608)

Resulta evidente, pues, que entender las cuestiones cuantitativas relativas a la FRECUENCIA (altas ventas), la INTENSIDAD (alta tensión) o, sencillamente, la ABUNDANCIA O ESCASEZ (altas dosis) a través de los conceptos físicos básicos ARRIBA/ALTO/SUBIDA O ABAJO/BAJO/BAJADA es un recurso cognitivo que convierte en concretas y fácilmente asimilables realidades que pueden presentar un cierto grado de complejidad.

\section{Propiedades y eventos}

Podemos dividir los usos metafóricos de alto y bajo que nos ocupan entre aquellos que se relacionan con propiedades y aquellos que se relacionan con eventos:

\section{I. Usos relacionados con las propiedades}

Es frecuente el vínculo entre alto y bajo con sustantivos abstractos que hacen referencia a propiedades: intensidad, resolución, mortalidad, cantidad ${ }^{10} \ldots$ Estas propiedades no deben entenderse ontológicamente como objetos y, por lo tanto, no pueden considerarse portadoras de (meta)propiedades:

On the basis of a greater range of linguistic data and with the support of some ancient and medieval philosophical views, I argue that such terms do not stand for objects, but rather for kinds of tropes, entities that do not have the status of objects, but only play a role as semantic values of terms and as arguments of predicates. Such 'non-objects' crucially differ from objects in that they are not potential bearers of properties. (Moltmann 2004: I)

El concepto de «tropo» empleado por Moltmann proviene de la metafísica y podemos decir, siguiendo a Villalba (2011: 91), que es «la realización concreta de una propiedad en un individuo». Dichas realizaciones concretas pueden darse en distintos grados, pero no contamos, al menos en español, con adjetivos precisos

10 Entendemos la CANTIDAD también como una propiedad. Asociaciones como altas cantidades o altas dosis hacen referencia al grado escalar cuantitativo en que se presentan objetos (altas dosis de estrógenos) substancias (altas dosis de arsénico) u otras realidades inmateriales (altas dosis de paciencia, altas dosis de amor). 
que, más allá de lo metafórico, puedan hacer referencia al grado (ni preciso ni aproximado) en que se dan"; una referencia directa (mucho/poco) o indirecta (alto/bajo, como explicábamos en el apartado 3) al concepto de CANTIDAD resulta siempre necesaria.

Hacemos referencia al concepto de GRADO, por ser precisamente su graduabilidad una de las características básicas de los adjetivos que nos ocupan: «Adjectives are gradable if they denote a property that can be present in an object to a greater or lesser degree» (Tribushinina 2008: 78).

De modo similar a cómo con el uso de percentiles se indica la posición exacta que tendría una entidad si su comparison class estuviese constituida por una clase ordenada de 100 elementos, creemos que en el empleo de un adjetivo dimensional también subyace un proceso de ubicación ${ }^{12}$ (aproximada) de una entidad dentro de una clase concreta que actúa como fondo de contraste.

Semantically, gradable adjectives can be informally defined as predicative expressions whose domains can be partially ordered according to some property that permits grading. For example, the domain of the adjective tall can be ordered according to a measure of height [...] (Kennedy 1999: XIII)

Estas representaciones serían las escalas. Los puntos o intervalos ordenados que las forman serían los grados.

El hecho de que estas escalas se conciban en un eje vertical ${ }^{13}$ puede ser de nuevo consecuencia (y causa, por retroalimentación) de la relación entre los conceptos de altura y cantidad tratada en el apartado 3.

\subsection{Usos relacionados con los eventos}

Aunque no resulta tan productiva como su asociación con sustantivos que expresan propiedades, los adjetivos alto y bajo pueden también relacionarse con nombres eventivos. En ese caso, el significado de los adjetivos que nos ocupa se

\footnotetext{
"Sí contamos, en ocasiones, con valores numéricos con los que expresar de formas precisas el grado en que se da una determinada propiedad en un objeto concreto: la resolución de la foto es de 1800 megapíxeles.

12 Empleamos ubicación y posición de manera metafórica, trasladando a lo espacial (a una escala) magnitudes abstractas $y$ valores numéricos.

13 Una prueba de esta proyección mental puede hallarse en el uso del vocablo nivel (un alto nivel de aceptación, el bajo nivel de las ventas...), relacionado, en principio, con la altura que alcanza, o con la altura a la que se ubica, una entidad. Es cierto que actualmente se emplea también con un sentido similar al de grado; sin un vínculo consciente con el concepto de VERTICALIDAD.
} 
vincula con el concepto de FRECUENCIA (ventas altas, alto abandono, contagios bajos...): es decir, con la mayor o menor repetición de un suceso.

Resulta evidente que, por ejemplo, las ventas de un producto no se acumulan sobre el suelo hasta alcanzar una determinada altura, pues se trata de eventos sin existencia física. La conexión entre esta idea de REPETICIÓN y el eje vertical ${ }^{14}$ se basa, de nuevo, en el proceso cognitivo al que hacíamos referencia en el apartado tercero.

En el apartado 5.2 mostraremos los criterios empleados en nuestra propuesta lexicográfica para agrupar o separar los usos de alto y bajo vinculados con propiedades y eventos. Mostraremos, además, algunos casos particulares que hemos considerado apropiado tratar de manera independiente.

\section{Explicación de la propuesta lexicográfica}

En este apartado daremos cuenta de los criterios adoptados a la hora de concretar la propuesta lexicográfica que se recoge en la parte final del artículo: trataremos de arrojar luz sobre las cuestiones lingüísticas que se han tenido en consideración e intentaremos justificar las decisiones lexicográficas (micro y macroestructurales) que se han adoptado durante su realización. Intentaremos, en definitiva, hacer explícitos los recursos empleados para conjugar la transparencia requerida por un diccionario con la complejidad de algunos de los conceptos manejados en los análisis semánticos de los apartados 2 y 3 .

\section{I. Estructura de la propuesta}

En nuestra propuesta lexicográfica abordaremos exclusivamente los sentidos de alto y bajo que se vinculan con los conceptos de CANTIDAD, INTENSIDAD y FRECUENCIA. La clasificación de los usos prototípicos de estos adjetivos (abordados en el apartado 2) quedará, pues, fuera del foco de esta parte de la investigación.

A pesar de que, en principio, es el empleo de alto y bajo como adjetivos lo que centra nuestra atención, recogeremos también usos adverbiales (muy similares a los adjetivales) con los que mostrar de forma más precisa el alcance léxico de las metáforas que nos ocupan.

Podemos decir, por lo tanto, que cada artículo estará encabezado por un lema (lema radical) y que, dentro de este, habrá una serie de subentradas

\footnotetext{
${ }^{14}$ La conexión entre el eje vertical y los eventos que nos ocupan puede detectarse, de modo similar a como hacíamos en la nota anterior, a través del uso del vocablo nivel: un nivel alto de ventas, un nivel bajo de abandono escolar...
} 
dependientes de sublemas (lemas categoriales) estructurados conforme al concepto de CLASE DE PALABRA.

A la hora de ordenar las entradas de cada artículo hemos optado por presentar en primer lugar los adjetivos, pues es el uso adjetival de estas palabras el que entendemos como prototípico dentro de su red polisémica.

\subsection{Separación y agrupamiento de las acepciones}

La cuestión de cómo determinar con precisión cuáles son los distintos significados que se pueden relacionar con una palabra dista mucho de estar resuelta en la lingüistica actual: «The trouble with word sense disambiguation is word senses (Kilgarriff 2006: 29): there is very little agreement about what word senses are» (Atkins y Rundell 2008: 269).

La elaboración de diccionarios tampoco cuenta, como consecuencia, con una metodología estandarizada a la hora de separar o agrupar acepciones: «After centuries of practical lexicography, there is still hardly any consensus on how to divide the semantics space of a lexical item» (Van der Meer 2006: 604).

Desde nuestra perspectiva resulta evidente, sin embargo, que los usos de alto y bajo que no se vinculan de forma directa con las dimensiones (o la ubicación) de un objeto merecen un tratamiento lexicográfico en el que estos aparezcan claramente diferenciados de los usos prototípicos. Los criterios empleados para la separación (o agrupación) de estos distintos sentidos metafóricos se tratarán con detalle en el apartado 5.4 .

\subsection{Las definiciones}

Cada una de las acepciones (o subacepciones) de nuestra propuesta lexicográfica presenta una definición que intenta conjugar la imprescindible claridad lexicográfica con la coherencia del tratamiento teórico ofrecido en la primera parte del artículo.

Las definiciones que empleamos responden a aquello que Seco (2003: 35) denomina definiciones impropias: «no son definiciones en metalengua del contenido, sino en metalengua del signo». Las definiciones, en estos casos, aportan información sobre el uso del signo, y son para Seco (2003, pág. 34) más explicaciones que verdaderas definiciones. Esto se mostraría en el hecho de que, en estos casos, lo definido no puede ser sustituido por la definición:

La sustitución de misericordioso por su definición académica, en un texto como Es misericordioso con los que sufren, nos daría este otro texto, en 
modo alguno equivalente: *Es dícese del que se conduele y lastima con los trabajos ajenos, con los que sufren. (Seco 2003: 35)

Así, en definiciones de adjetivos que, como las nuestras, se inician con dícese - aplícase, solo el elemento que funciona como especificador del nombre (en nuestro caso, aquello) es «intercambiable por la palabra-entrada» (Seco 2003: 36). A pesar de que comprendemos y valoramos la aportación teórica de Seco respecto a la naturaleza propia o impropia de esta clase de definiciones, creemos que estas responden a una fórmula convencionalizada ${ }^{15}$ lo suficientemente clara como para que el hecho de que la definición en su conjunto no sea un sustituto válido de la palabra definida no suponga un problema de interpretación.

Los ejemplos empleados para acompañar las definiciones de la propuesta lexicográfica han sido tomados ( $y$, en ocasiones, adaptados, para facilitar su comprensión) fundamentalmente del Corpus de Referencia del Español Actual.

\section{5..3.I. «Lo normal o adecuado»}

En las definiciones de nuestra propuesta lexicográfica empleamos los conceptos de LO NORMAL y LO ADECUADO como referencias para establecer si algo puede considerarse alto o bajo. Para entender este recurso descriptivo debemos explicar primero cómo se calcula el valor cuantitativo de los adjetivos dimensionales cuando estos se emplean con sus sentidos prototípicos en los intercambios comunicativos reales.

Los adjetivos dimensionales no poseen un valor cuantitativo fijo (alto, por ejemplo, no se puede asociar a una determinada medida de manera general), sino que este varía según las circunstancias comunicativas en que aparezcan: en circunstancias normales, el valor de alto es muy distinto cuando se habla, por ejemplo, de un niño alto y de un edificio alto.

Para saber qué magnitudes se deben asociar con un adjetivo, es necesario, en primer lugar, poner en relación la entidad descrita por este con una determinada categoría de cosas. La categoría concreta respecto a la que se debe relativizar el valor de un adjetivo subsectivo es lo que tradicionalmente se ha denominado comparison class: «a comparison class is a subset of the universe of discourse which is picked out relative to a context of use» (Klein 1980: 13).

Solo en relación a dicha clase podrá empezar a darse 'forma' al valor cuantitativo de un adjetivo dimensional. Así, el valor de alto en Un niño de 8 años

\footnotetext{
15 Seco señala que esta clase de definiciones 'irregulares' son frecuentes «en un amplio sector de la lexicografía, dentro del cual figuran todos los diccionarios españoles, encabezados por el de la Academia» (Seco 2003: 34).
} 
muy alto podrá ser muy distinto dependiendo de si se relativiza la altura respecto a la clase de [los niños de 8 años] o, en general, respecto a la de [las personas]: que un niño midiera $1,70 \mathrm{~m}$ sería más que suficiente para que este fuera considerado alto como niño; debería, sin embargo, medir algo más para que se le pudiera considerar alto como persona. Esta propiedad de los adjetivos se conoce como subsectividad: los niños altos son un subconjunto (subset, en inglés) de la categoría con que se comparan, y el valor cuantitativo del adjetivo depende de las características del conjunto del que forman parte.

Las entidades suelen ser miembros de infinitas categorías simultáneamente, pero en cada enunciado hay una única clase de comparación 'válida': si el emisor y receptor no relativizan respecto a la misma clase, el valor cuantitativo con que relacionen un adjetivo dimensional en un contexto determinado podrá ser muy distinto en cada caso. Como se señala en Ynduráin (2019: 6), la búsqueda de la Relevancia es el factor que actúa como guía inferencial y posibilita la selección de la categoría de contraste adecuada.

A pesar de que este primer paso resulta imprescindible para aproximarse al valor cuantitativo de un adjetivo dimensional, no es, sin embargo, suficiente. Hay una segunda cuestión que debe ser resuelta: iqué implica, por ejemplo, pertenecer a la clase de [los altos] dentro de la categoría de [los niños]?

Desde nuestra perspectiva, el uso de estos adjetivos implica una comparación entre las propiedades dimensionales de la entidad sobre la que se predica una propiedad dimensional y la clase de cosas que actúa como referencia. El problema reside en cómo se establece la comparación con dicho conjunto:

How [...] can we express a positive adjective in terms of comparative? There are several alternatives: $a$ is tall can be rendered as either $a$ is taller than most (Langford) or $a$ is taller than one would expect (Wierzbicka) or else $a$ is tall to $a$ degree $d$ and $d$ is towards the top of the scale for tallness (Creswell). (Paoli 1999: 7I)

A number of linguists have suggested that the form of the positive degree of relative adjectives signifies a greater than average, or greater than norm (or smaller than average norm) value on the dimensional-scale relevant to the adjective in question. (Rusiecki 1985: 28)

Consideramos que el prototipo de una categoría es la entidad que actúa como referencia y permite establecer si los elementos de dicha categoría son, por ejemplo, grandes, pequeños o medianos. No cualquier elemento distinto del prototipo debe ser necesariamente considerado susceptible de describirse mediante un adjetivo dimensional: en torno a este prototipo existe una zona neutra que recoge todo elemento dotado de la propiedad que se esté evocando en un 
grado irrelevantemente distinto al grado en que esta propiedad se presente en ese prototipo.

En la figura I se ilustra esta idea a través de una escala (relativa al tamaño) en la que aparece el prototipo de una categoría, la zona neutra y las partes de la escala que se relacionan con cada uno de los antónimos polares (grande y pequeño, en este caso).

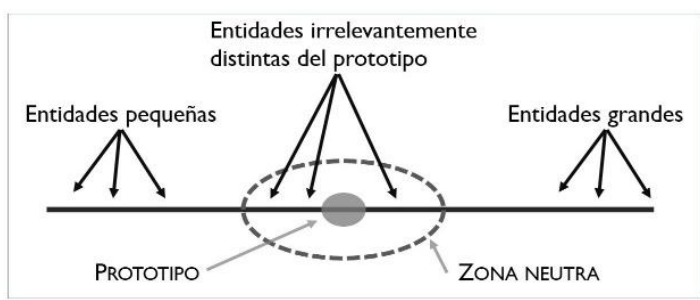

Figura I - Prototipo y zona neutra

Los elementos que se muestren como irrelevantemente distintos del prototipo compartido (en el aspecto dimensional que se esté tratando) formarán parte, junto al mismo prototipo, de ese espacio denominado zona neutra. De los elementos que destaquen por determinada diferencia dimensional (que en un contexto específico sí sea relevante) se podrá afirmar que no son 'neutros' (dentro de su categoría) y será posible calificarlos con uno de los adjetivos polares.

Podemos decir, por lo tanto, que $X$ es alto será un enunciado verdadero siempre que $X$ sea relevantemente más alto que el prototipo de la categoría que actúe como comparison class.

En contextos no marcados, creemos que una diferencia dimensional respecto a un prototipo es relevante cuando resulta perceptualmente evidente: solo cuando el estímulo (empleando la terminología de Rosch [1975: 532]) se muestre de manera clara a los sentidos, el hablante calificará dicha entidad con un adjetivo dimensional. Desde la perspectiva del oyente, si no hay una relevancia práctica concreta, este, ante la presencia de un adjetivo dimensional, interpretará que hay una intención comunicativa de carácter descriptivo, es decir, que la diferencia dimensional del objeto respecto al prototipo será una diferencia sensorialmente evidente $y$, por lo tanto, descriptivamente relevante. Así, del mismo modo que resultaría redundante hablar de una mesa con patas, debido a que el tener patas es una propiedad prototípicamente asociada a mesa, resultaría innecesario también hablar de una mesa de tamaño normal si el contexto comunicativo no exige esa especificación: es, precisamente, una mesa de tamaño normal (que se ubicaría dentro de la zona neutra) lo que evoca el sustantivo mesa por sí solo. Cuando una 
mesa no sea (dimensionalmente) 'normal', entonces sí resultará descriptivamente enriquecedor recurrir a un adjetivo dimensional para referirse a ella.

Son, por lo tanto, los efectos cognitivos del enunciado (el interés que puede suponer la información sobre la excepcionalidad dimensional del elemento del que se habla) lo que subyace a la inferencia de cuál debe entenderse que es la diferencia dimensional de una identidad respecto al prototipo de su clase de comparación en cada situación comunicativa concreta.

Cuando nos encontramos ante usos metafóricos como los que nos ocupan, el mecanismo inferencial es muy similar: las ventas de un determinado modelo de vehículo son altas cuando, en relación con una determinada categoría (los vehículos de precio similar, los vehículos de tamaño similar, el mismo vehículo en etapas anteriores o posteriores...), estos eventos superan de forma relevante (significativa) el número de repeticiones que se considera 'normal'.

En nuestra propuesta lexicográfica hemos optado por no hacer mención de manera explícita a la necesidad de relativizar respecto a una CLASE DE REFERENCIA: Consideramos que el concepto de NORMALIDAD al que nos referimos en las definiciones se establece siempre de manera intuitiva respecto a un determinado conjunto de entidades que actúa como fondo de contraste.

Además, entendemos que las ideas de PROTOTIPO y ZONA NEUTRA aparecen recogidas también a través de dicha referencia a «lo normal» ${ }^{16}$ : de manera natural e intuitiva, la idea de NORMALIDAD recoge la de PROTOTIPICIDAD y, por su vaguedad inherente, admite, además, las desviaciones respecto al prototipo que se entiendan como irrelevantes ${ }^{17}$ en un contexto dado.

Podemos decir, pues, que la propia vaguedad del concepto de NORMALIDAD pone en manos de la competencia pragmática de los usuarios de la lengua la relación de lo dimensional con una clase de entidades y con un determinado grado de desviación respecto a algún tipo de norma: «Como existe una zona de

${ }^{16}$ En algunos diccionarios sí aparece de manera más o menos explícita el concepto de CLASE DE REFERENCIA: se indica que se debe relativizar respecto a aquello «que forma serie con el nombrado» en el Diccionario del Español Actual (20I5, s. v. grande), y se dice que una cosa es pequeña cuando lo es «en comparación con otras de su misma especie» en el DRAE (200l, s. v. pequeño). Mientras, la PROTOTIPICIDAD y la NORMALIDAD se suelen expresar mediante el siguiente tipo de conceptos: «lo normal o lo corriente», en el Diccionario del Español Actual (20I5, s. v. grande, pequeño y largo), «misura ordinaria», en Lo Zingarelli (20I5, s. v. grande); «average size», en Longman (2009, s. v. big); y «moyenne [taille]», en Le Petit Robert (2012, s. v. grand).

${ }_{17}$ A pesar de que, como se recoge en Ynduráin (2019: 2), consideramos imprescindible recurrir al concepto de RELEVANCIA para explicar el funcionamiento de esta clase de adjetivos, creemos que, por su complejidad, las referencias explícitas a dicho concepto no tienen cabida en las definiciones de un diccionario no especializado. 
indeterminación para su aplicación, los adjetivos graduables son vagos e implícitamente comparativos» (Di Tullio 2010: 193) ${ }^{18}$.

Además, hemos optado por añadir una referencia a «lo adecuado», ya que, en ocasiones, el prototipo presenta un marcado carácter práctico o existe algún tipo de referencia explícita que determina cuáles 'deben' ser las dimensiones de una entidad o cuál 'debe' ser el número adecuado de repeticiones para un evento relacionado con ella. En dichos casos, el uso de adjetivos dimensionales posee un valor que va más allá de lo perceptivo y del mero enriquecimiento descriptivo: a veces un objeto es pequeño porque lo es para un determinado fin y cualquier diferencia (independientemente de cómo de claramente sea percibida) respecto al prototipo resulta relevante. Ebeling y Gelman (1994: II79) señalan, acerca del uso de los adjetivos big y small, que, en una de sus posibles interpretaciones, "the object is judged with regard to its intended use». Así, un enunciado como The hat is big puede tener, entre otras, una interpretación de carácter funcional: «e.g. too big for a tiny dollı. Del mismo modo, la intensidad de una corriente eléctrica puede ser definida como baja si esta es inferior a lo que resultaría adecuado para hacer funcionar un determinado aparato.

\subsection{Cuestiones relativas a cada acepción}

\subsection{Adjetivos}

La primera acepción que recogemos de alto y bajo en nuestra propuesta lexicográfica (apartado 6) subsume bajo una misma definición los casos en los que los adjetivos que nos ocupan se relacionan con propiedades (4.I) y aquellos en que se relacionan con eventos (4.2).

En el caso de que hubiéramos optado por tratar por separado cada uno de los dos usos señalados podríamos haber empleado las siguientes definiciones:

I. Se aplica a propiedades cuando se dan en un grado mayor/menor de lo normal o adecuado.

\footnotetext{
18 Conviene, por otro lado, señalar que nuestra propuesta no se recogen todas las estructuras lingüísticas en las que pueden aparecer adjetivos dimensionales. Así, en un enunciado como Mi hermano es más alto que mi hermana, aunque ambos son muy bajos, el sentido de alto no se corresponde con el de algo que presenta una altura «mayor de lo normal o adecuado». Consideramos que los adjetivos dimensionales, en este aspecto, se integran de manera natural en los conocimientos lingüísticos generales de los hablantes: a estos corresponde el cohonestar lo que saben de su idioma con los rasgos aportados por una (siempre limitada) explicación lexicográfica. Lo mismo ocurre cuando uno de los adjetivos tratados aparece acompañado de expresiones de grado: en la interpretación de alto en muy alto debe integrarse la definición correspondiente con la competencia lingüística y comunicativa que se presupone a los hablantes.
} 
2. Se aplica a eventos cuando son más/menos frecuentes de lo normal o adecuado.

Hemos considerado más oportuno, sin embargo, recurrir de modo general a aquello para recoger, bajo esta expresión abierta, tanto las propiedades como los eventos. Entendemos, por un lado, que la distinción ontológica entre eventos y propiedades no resulta necesaria en un diccionario no especializado y, por otro, que los adjetivos intenso y abundante, que aparecen también en la definición, ya conectan con estas ideas de un modo que resulta más intuitivo para el usuario de obras lexicográficas.

Hemos considerado necesario diferenciar una subacepción en la que los adjetivos que nos ocupan se relacionan lingüísticamente con nombres de realidades que implican una emisión sonora; en estos casos los adjetivos señalan, por un proceso metonímico evidente, cuál es el grado en que se da el volumen de dicha emisión: en música alta se activa mentalmente el concepto de VOLUMEN (SONORO) y se entiende que la música en cuestión se presenta con un determinado grado de dicha propiedad. Lo mismo ocurre cuando los sustantivos aluden a eventos (sonoros): un grito alto es un grito cuyo volumen se presenta en un grado superior a lo normal o adecuado. En la propuesta recogemos los eventos y otras realidades empleando de nuevo la expresión ontológicamente abierta aquello. Esta subacepción cuenta, como veremos más adelante, con un uso adverbial análogo.

Los usos relativos al tono de un sonido también aparecen en nuestra propuesta como acepciones diferenciadas. Bosque (1985: 68) señala que en las lenguas latinas es frecuente el uso de adjetivos que vinculan el tono (grave o agudo) con el eje vertical.

A pesar de que el concepto de TONO permite recuperar la propiedad FRECUENCIA DE VIBRACIÓN DE ONDA (un sonido con mucha frecuencia de vibración es agudo), el origen del uso de alto y bajo para hacer referencia al tono probablemente se encuentra en un proceso sinestésico que lleva a percibir lo agudo como ligero (y más susceptible de estar arriba) y lo grave como pesado ( $y$ más susceptible de estar abajo) ${ }^{19}$.

No estamos, pues, ante significados de alto y bajo cuyo desarrollo metafórico los vincule con los conceptos de CANTIDAD o de FRECUENCIA; los detalles sobre el origen de su significado quedan fuera, por lo tanto, del objetivo de nuestra investigación. Creemos, sin embargo, que resulta apropiado incluir estas acepciones

${ }^{19}$ Para el origen de los términos grave, leve y agudo en español, véase Corominas y Pascual [ 1981 - 1991] $(2012$, s v. grave, s. v. leve y s. v. agudo). 
tonales en nuestra propuesta para dejar constancia, precisamente, de su carácter particular y abrir el camino para futuras investigaciones ${ }^{20}$.

Por un proceso metonímico bastante reconocible resulta frecuente también encontrar referencias a instrumentos o cantantes altos y bajos, dependiendo de si el sonido que estos producen es agudo o grave en relación a otros con los que forman serie. Este sentido de los adjetivos se recogerá en una subacepción en la que se hace referencia directa a su origen metonímico: «por extensión...».

\subsubsection{Adverbios}

Las dos acepciones adverbiales que recogemos están relacionadas con el sonido. El primer uso indica el volumen al que se realiza una acción. De nuevo, la referencia a que estos adverbios se aplican a acciones se halla más en el verbo que empleamos (realizar) que en el pronombre (aquello) utilizado. En el segundo uso, de nuevo asimilamos lo alto con lo agudo y lo bajo con lo grave.

\section{Propuesta lexicográfica \\ Alto/a (ADJETIVO)}

I Se aplica a aquello que es más intenso o abundante de lo normal o adecuado. Los ejercicios de resistencia de alta intensidad pueden ayudar a hombres y mujeres. Mars Express envió las primeras imágenes de alta resolución de la superficie de Marte. Enciende la música a un volumen alto y a veces grita mientras pinta. Su más reciente producción alcanzó altas ventas.

I.b Se aplica a aquello que presenta un grado de volumen sonoro mayor de lo normal o adecuado. Detestaba la música alta, el ruido y el humo. Se oyó, de pronto, una voz muy alta, al otro lado de la fuente. Uno de los corredores da un grito alto, lleno, casi musical.

2 Se aplica a una emisión sonora cuando es aguda, es decir, cuando produce muchas vibraciones por segundo. Es una obra dificil de interpretar por sus notas altas.

2.b Por extensión, se aplica a la fuente sonora que produce sonidos más agudos que los de otras fuentes sonoras de la misma clase. En todos los cortes Paul Desmond toca el saxo alto con su sonido fluido y elevado.

\footnotetext{
${ }^{20}$ En la definición hemos decidido no abordar la cuestión de si aquello que consideramos que es agudo lo es respecto a una clase de referencia o si es una propiedad de carácter absoluto que depende de nuestro sistema auditivo. Nos limitamos a señalar que alto y bajo resultan equivalentes a agudo y grave, respectivamente. Del mismo modo, señalamos que las vibraciones por segundo son «muchas» o «pocas» sin entrar a valorar cómo deben interpretarse esos cuantificadores en el contexto concreto que nos ocupa.
} 


\section{Alto (ADVERBIO)}

I Se aplica a aquello que se realiza con un grado de volumen sonoro mayor de lo normal o adecuado. Se hablaban alto, separados por los transeúntes.

2 Se aplica a aquello que se realiza en un tono agudo. Los rangos vocales varían ampliamente entre individuos $y$, en general, las mujeres pueden cantar más alto que los hombres.

\section{Bajola (ADJETIVO)}

I Se aplica a aquello que es menos intenso o abundante de lo normal o adecuado. Azerbaiyán mantiene una guerra de baja intensidad con su vecina Armenia desde 1989. Las fotografias obtenidas del planeta son de baja resolución. Hay que mantener el radiocasete con un volumen bajo. Tuvieron que cerrar sus puertas ante las bajas ventas reportadas.

I.b Se aplica a aquello que presenta un grado de volumen sonoro menor de lo normal o adecuado. La luz era escasa y la música muy baja. Habló doña Inés con una voz baja y agradable. Estos perros usan un gemido bajo y murmurante para llamar la atención.

2 Se aplica a una emisión sonora cuando es grave, es decir, cuando produce pocas vibraciones por segundo. Había rumores roncos en las notas bajas del piano.

2.b Por extensión, se aplica a la fuente sonora que produce sonidos más graves que los de otras fuentes sonoras de la misma clase. Se presentaron con con Frank Dubuy en el trombón bajo y Ralph Pottle en el corno francés.

\section{Bajo (ADVERBIO)}

I Se aplica a aquello que se realiza con un grado de volumen sonoro menos de lo normal o adecuado. Había mesas ocupadas: parejas anodinas que hablaban bajo y sorbían el té.

2 Se aplica a aquello que se realiza en un tono grave. Axel puede cantar más bajo que Barry White.

\section{Conclusión}

La red polisémica que presentan en torno a sus significados prototípicos los adjetivos alto y bajo cuenta con una serie de casos en los que estas palabras se emplean para expresar el grado con que se presentan las propiedades (alta mortalidad, baja resolución) o la frecuencia con que se produce un evento (ventas altas, bajos contagios). 
En ambos usos subyace la misma metáfora cognitiva: la identificación conceptual de ARRIBA con MUCHO y de ABAJO con POCO, independientemente del tipo de entidades (físicas o abstractas) de las que se esté predicando algo.

La base de esta metáfora se encuentra en una experiencia recurrente dentro del contexto físico en el que vivimos: es frecuente que el aumento de la presencia de una substancia o de una serie de objetos en un lugar determinado conlleve la ocupación de mayor espacio vertical por parte de estos. Esta asociación resulta cognitivamente útil $y$, por ello, lingüísticamente productiva; en el español, además de los casos ya mencionados, encontramos numerosas expresiones que pueden explicarse a través del fenómeno señalado: los precios suben, los salarios bajan, un montón de tiempo...

Consideramos que el tratamiento lexicográfico de los adjetivos que nos ocupan debe recoger estos sentidos, junto a aquellos que se relacionan de forma indirecta con el fenómeno semántico previamente abordado. Nuestra propuesta puede servir de base para la tarea de convertir en discretos y mutuamente excluyentes el conjunto de significados que presentan estas formas léxicas: «Dictionaries greatly exaggerate the measure of discreteness of meanings, and are inclined to set clear-cut borders where a closer examination... reveals only a vague intermediate area of overlapping meanings» (Apresjan 1973: 9)

Entendemos, además, que nuestra presentación de los significados relativos al concepto de CANTIDAD como traslaciones surgidas a partir de un significado central o prototípico permite superar, como se busca desde aproximaciones cognitivistas, la linealidad tradicional de los diccionarios:

\footnotetext{
Una microestructura desde el modelo cognitivo se plantearía como la organización de los significados de una palabra en torno a redes radiales que tuvieran diferentes niveles semánticos unidos por medio de mecanismos cognitivos como la metáfora o la metonimia [...]. (Ibarretxe-Antuñano 20l0: 200)
}

\section{Referencias bibliográficas}

APRESJAN, J. D. (1973). Regular Polysemy. Linguistics, I42, 5-39.

AtKInS, B. T. S. Y M. RUNDelL. (2008). The Oxford Guide to Practical Lexicography. Nueva York: Oxford University Press.

Barcelona, A. (20I2). La metonimia conceptual. En: Ibarretxe-Antuñano, I. y Valenzuela, J. (dirs.), Lingüistica cognitiva. Barcelona: Anthropos, I23-156.

BENCZES, R. (2006). Creative Compounding in English. The Semantics of Metaphorical and Metonymical Noun-Noun Combinations. Ámsterdam: John Benjamins Publishing Company. 
Bosque, I. (1985). Usos figurados de los adjetivos que denotan dimensiones físicas. En: Alvar López, M. (hom.), Philologica hispaniensia: in honorem Manuel Alvar (Vol. 2). Madrid: Gredos, pp. 63-80.

Clark, H. H. (1973). Space, time, semantics, and the child. En: Moore, T. E. (ed.), Cognitive Development and the Acquisition of Language. Nueva York $y$ Londres: Academic Press, 27-63.

COROMINAS, J. Y PASCUAL, J. A. [1981-199I] (2012). Diccionario crítico etimológico castellano e hispánico. Madrid: Gredos [edición electrónica].

CORRAles Zumbado, C. (1977). El campo semántico «dimensión» en español. Santa Cruz de Tenerife: Publicaciones del Aula de Cultura del Excelentísimo Cabildo Insular de Santa Cruz de Tenerife.

DI Tullio, A. (2010) [2005]. Manual de gramática del español. Buenos Aires: Waldhuter.

Diccionario de la lengua española. Véase: REAL ACADEMIA ESPAÑOLA Y AsOCIACIÓN DE ACADEMIAS DE LA LENGUA ESPAÑOLA (200I).

Diccionario del español actual. Véase: SECO, M., RAMOS, G. Y ANDRÉS, O. (1999).

Dirven, R. y Taylor, J. (1988). Conceptualization of vertical space in English: The case of Tall. En: Rudzka-Ostyn, B. (ed.) Topics in Cognitive Linguistics. Ámsterdam: John Benjamins Publishing Company, 379-402.

EbeLING, K. S., y GeLmAN, S. A. (1994). Children's use of context in interpreting «big» and «little». Child Development, 65(4), II78-II 92.

Geckeler, H. (1997). Réflexions sur le champ lexical adjectival des dimensions spatiales du français. En: Montibus, M. J. (ed.), L'Organisation lexicale et cognitive des dimensions spatiales et temporelles. Actes d'EUROSEM 1996. Reims: Presses Universitaires de Reims, 95-106.

GRADY, J. E. (2005). Primary metaphors as inputs to conceptual integration. Journal of Pragmatics, 37, 1595-1614.

IBARRETXE-ANTUÑANO, I. (2010). Lexicografía y Lingüística Cognitiva. Revista Española de lingüística aplicada, 23, I95-2। 4.

KENNEDY, C. (1999). Gradable adjectives denote measure functions, not partial functions. Studies in the Linguistic Sciences, 29(I), 65-80.

Kilgarriff, A. (2006). Word senses. En: Agirreand, E. y Edmonds, P. (eds.), Word Sense Disambiguation: Algorithms and Applications. Nueva York: Springer, New York, 29-46.

KLEIN, E. (1980). A Semantics for Positive and Comparative Adjectives. Linguistics and Philosophy, 4, I-45.

KOVECSES, Z. (20I5). Where Metaphors Come From: Reconsidering Context in Metaphor. Oxford: Oxford University Press.

LAKOFF, G. Y JOHNSON, M. (1980). Metaphors we live by. Chicago: The University of Chicago Press. 
Lakoff, G. (1993). The Contemporary Theory of Metaphor. En: Ortony, A. (ed.), Metaphor and Thought. Cambridge: Cambridge University Press, 202-25I.

LANGACKER, R. W. (1987). Foundations of Cognitive Grammar, vol.l: Theoretical prerequisites. Palo Alto: Stanford University Press.

LANGACKER, R. W. (1993). Reference-point constructions. Cognitive Linguistics, 4, I38).

LANGACKER, R. W. (1995). Raising and Transparency, Language, 7I.I, I-62.

LEVINSON, S. C. (2003). Space in Language and Cognition, Explorations in Cognitive Diversity. Cambridge: Cambridge University Press.

LINDE-USIEKNIEWICZ, J. (2000). Określenia wymiarów w języku polskim. Varsovia: Wydział Polonistyki Uniwersytetu Warszawskiego.

Lo Zingarelli 20I5. Vocabolario della lingua italiana. Véase: ZINGARELLI, N. (20I5).

Longman Dictionary of Contemporary English. Véase: MAYOR, M. (2009)

MAYOR, M. (2009). Longman Dictionary of Contemporary English. Londres: Longman Publishing Group.

Petit Robert 2012 (20I I). OBRA COLECTIVA. Paris: Le Robert.

Moltmann, Friederike (2004). Properties and Kinds of Tropes: New Linguistic Facts and Old Philosophical Insights. Mind, I I 3 (449), I-4I.

MULIER, U. (2009) Análisis semántico - sintáctico de cuatro adjetivos de dimensión: alto, bajo, largo y corto. (Tesina dirigida por Renata Enghels). Gante: Universiteit Gent. Faculteit Letteren en Wijsbegeerte.

Radden, G. y Kövecses, Z. (1999). Towards a theory of metonymy. En: Panther, K. U. y Radden, G. (eds.), Metonymy in Language and Though. Amsterdam: John Benjamins Publishing Company, 17-59.

Real Academia Española. Corpus de referencia del español actual [en línea] [consultado el I de septiembre de 2020]. Disponible en Web:

< http://corpus.rae.es/creanet.html>

RAKHILINA, E. V. (2000). Kognitivnyj analiz predmetnyx imen: semantika i sočetaemost'. Moscú: Russkie slovari.

RosCH, E. (1975). Cognitive reference points. Cognitive Psychology, 7, 532-47.

Rusiecki, J. (1985). Adjectives and Comparison in English: a Semantic Study. Londres: Longman.

SANtos DomíngueZ, L. A. y Espinosa ElORZA, R. Mª. (1996). Manual de Semántica Histórica. Madrid: Síntesis.

Schmid, H. J. (2007). Entrenchment, Salience, and basic levels. En: Geeraerts, D. y Cuyckens, H. (eds.), The Oxford Handbook of Cognitive Linguistics. Oxford: Oxford University Press, II7-138.

SECO, M., RAMOS, G. Y ANDRÉS, O. (1999). Diccionario del español actual. Madrid: Aguilar.

SECO, M. (2003). Estudios de lexicografia española. Madrid: Gredos. 
TRIBUSHININA, E. (2008). Cognitive reference points. Semantics beyond the prototypes in adjectives of space and colour. Utrecht: LOT.

Tyler, A. Y Evans, V. (2003). The Semantics of English Prepositions. Spatial Scenes, Embodied Meaning and Cognition. Cambridge: Cambridge University Press.

VAN DeR Meer, G. (2006). It's about Time: On Coherence and Simplicity in Dictionary Entries. English Studies, 87, 602-616.

Villalba, X. (201 I). Las nominalizaciones deadjetivales con lo y las cualidades. En: Escandell, M. V., Leonetti, M. y Sánchez, M. C. (coords.), 60 problemas de gramática: dedicados a Ignacio Bosque. Madrid: Akal, 90-95.

Vogel, A. (2004). Swedish Dimensional Adjectives. Stockholm studies in Scandinavian philology, 36. Estocolmo: Acta Universitatis Stockholmensis.

YNDURÁIN, C. (20I5). Los adjetivos dimensionales en español: Análisis semántico y propuesta lexicográfica. (Tesis de Doctorado). Madrid: Universidad Autónoma de Madrid.

YNDURÁIN, C. (2019). El cálculo del valor cuantitativo de los adjetivos dimensionales. Diálogo de la lengua, II, I- I4.

ZingArelLI, N. (20I5). Lo Zingarelli 20I5. Vocabolario della lingua italiana. Bolonia: Zanichelli. 